\title{
Novel Approach to the Quantitation of Regional Left Ventricular Systolic and Diastolic Function Using Tissue Doppler Imaging to Create a Myocardial Velocity Profile and Gradient
}

\author{
Sayuri Fujimoto, MD; Takashi Oki, MD; Tomotsugu Tabata, MD*; Hideji Tanaka, MD*; \\ Hirotsugu Yamada, MD*; Yoshifumi Oishi, MD; Takeo Ishimoto, MD; \\ Susumu Ito, MD*; Yasuhiko Abe**; Ryoichi Kanda**
}

\begin{abstract}
The myocardial velocity profile (MVP) and gradient (MVG) between the endocardium and epicardium of the left ventricular (LV) wall measured by color-coded tissue Doppler imaging (TDI) are new indices for evaluating regional LV myocardial function. However, accurate recording and measurement of the MVP is difficult using conventional methodology because of the stochastic nature of the ultrasound signal; that is, the effect of speckled noise. The aim of this study was to validate the accuracy and establish the validity of a newly developed method for measuring the MVP and MVG using 10 clinically normal controls and 10 patients with a hypertensive hypertrophied LV posterior wall. A non-isotropic, averaging algorithm was developed that was capable of obtaining a stable MVP (averaged MVP). Averaged MVP was recorded using parasternal, LV short-axis, color-coded TDI, placing regions of interest along the $\mathrm{LV}$ posterior wall with the reference point for angle-correction being at the center of LV contraction. The velocity from epicardium to endocardium within the region of interest was automatically angle-corrected to calculate the velocity component radially relative to the LV cavity and was spatially averaged along the circumference within the region of interest. Inter- and intraobserber variabilities of measurements were lower in the averaged MVP and MVG than in the conventional MVP and MVG. The correlation coefficients of the linear regression lines of systolic and early diastolic MVPs in the LV posterior wall were higher in all controls and hypertensive patients with the averaged method than with the conventional TDI procedures. The mean peak systolic and early diastolic MVGs were lower in the hypertensive group than in the controls. In conclusion, the newly developed averaged MVP provides a stable and reproducible index for the quantitative assessment of regional LV myocardial function. (Circ J 2003; 67: 416-422)
\end{abstract}

Key Words: Left ventricular hypertrophy; Left ventricular wall motion; Myocardial velocity gradient; Myocardial velocity profile; Tissue Doppler imaging

$\mathbf{T}$ issue Doppler imaging (TDI) facilitates the quantitative evaluation of regional left ventricular (LV) myocardial motion velocity ${ }^{1,2}$ and is used clinically to assess LV function in heart disease. Although the effect of the overall heart motion and changes in target sample volumes during a cardiac cycle introduce errors, pulsed TDI has many advantages, such as excellent time resolution and the capacity to record the peak cardiac wall motion velocity during the different phases of the cardiac cycle, thereby serving as an index that is unaffected by preload ${ }^{3-6}$ Colorcoded TDI provides an instantaneous measurement of regional myocardial lengthening or shortening velocity per unit length, the myocardial velocity gradient (MVG), and it has been reported that the MVG can be used as a parameter

(Received August 5, 2002; revised manuscript received February 12, 2003; accepted February 21, 2003)

Cardiovascular Section, National Higashi Tokushima Hospital, Tokushima, *The Second Department of Internal Medicine, School of Medicine, The University of Tokushima, Tokushima and **Toshiba Corporation, Medical Systems Research and Development Center, Tochigi, Japan

Mailing address: Takashi Oki, MD, Cardiovascular Section, National Higashi Tokushima Hospital, 1-1 Ohmukai-kita, Ohtera, Itano, Itano, Tokushima 779-0193, Japan

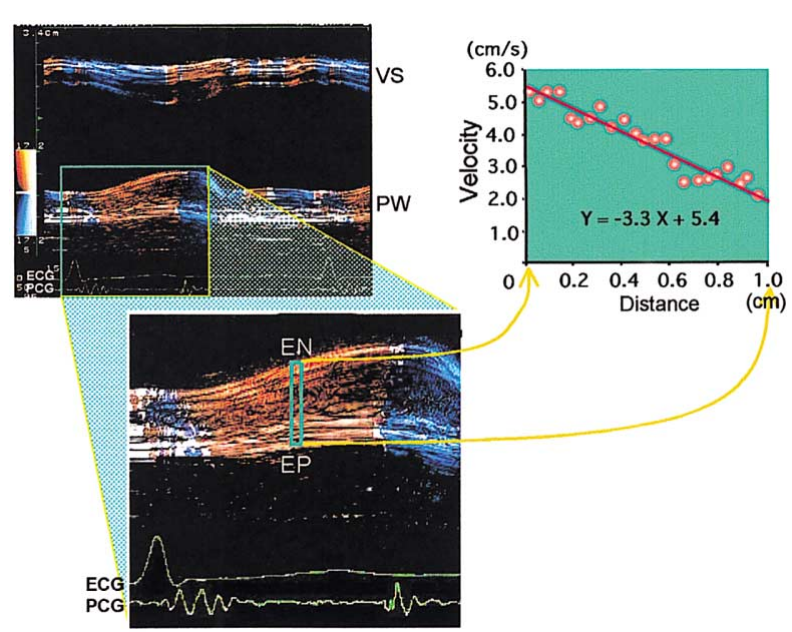

Fig 1. Measurement of the myocardial velocity gradient of the left ventricular posterior wall (PW) at mid-systole obtained from the color-coded M-mode tissue Doppler imaging using the conventional method. The myocardial velocity profile between the endocardium (EN) and epicardium (EP) of the posterior wall at mid-systole is extracted (Left), and the slope in linear regression of the profile represents the myocardial velocity gradient (Right). VS, ventricular septum. 

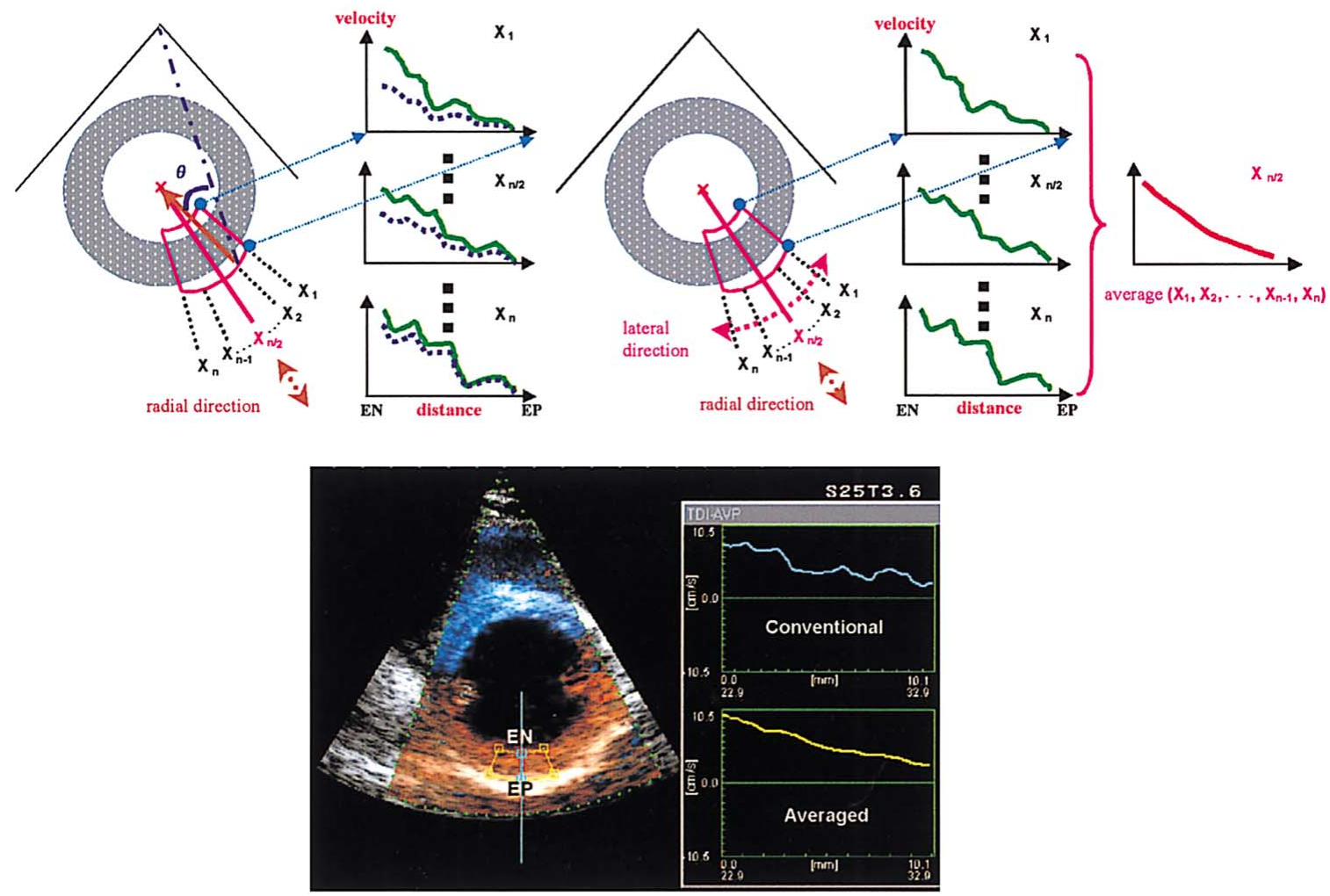

Fig 2. Method of recording the averaged myocardial velocity profile of the left ventricular posterior wall. First, after setting the hypothetical center of left ventricular (LV) contraction $(X)$, the velocity data were corrected by the actual Doppler angle $\theta$ ) of incidence for each pixel in the radial direction (Top and Left). A 2-D region of interest (ROI) was set along the LV posterior wall with the reference point for angle-correction being at the center of LV contraction. The velocities at each point within the ROI were automatically angle-corrected to calculate the velocity component radial to the LV cavity. In the velocity-distance relationship (myocardial velocity profile curve) shown in the Top and Left panel, the broken lines (blue) represent the observed velocity distribution from lines $\mathrm{X}_{1}$ to $\mathrm{Xn}$, and the solid lines (green) represent the angle-corrected velocity distribution for each radial direction ( $\mathrm{X}_{1}$ to $\mathrm{Xn}$ ) in the ROI. Next, the velocities of $\mathrm{X}_{1}$ to $\mathrm{Xn}_{\mathrm{n}}$ were averaged along the lateral direction (Top and Right). To improve the stability and accuracy of the velocity profile measurement, the myocardial velocities after angle-correction were spatially averaged along the circumference of the LV short axis images. The lateral average of the velocity profile (orange) at the center of the ROI (line Xn/2) was obtained by taking the average of the angle-corrected velocities at the same radial distance along lines $\mathrm{X}_{1}$ to Xn. (Bottom) Actual measurement of the myocardial velocity profile using conventional (blue) and averaged (yellow) methods. EN, endocardium; EP, epicardium.

that excludes the effect of overall heart motion and preload7-9 However, the MVG is calculated on the basis of the hypothesis that systolic and diastolic velocity in the ventricular septum from the endocardium of the right ventricular side to the endocardium of the LV side or LV posterior wall from the endocardium to the epicardium changes linearly. Furthermore, sampling at limited points during each cardiac cycle introduces problems of stability and reproducibility into the recording, as well as cyclic variation of integrated backscatter ${ }^{10}$ It has also been established that the velocity distribution in the hypertrophied myocardium is not always linear in patients with hypertrophic cardiomyopathy or cardiac amyloidosis?,11

We have developed novel TDI software that can record the myocardial velocity profile (MVP) and MVG to characterize LV myocardial function, ${ }^{11,12}$ and the goal of this study was to demonstrate that measurement of systolic and early diastolic MVP and MVG in the LV myocardium in normal and in hypertensive hypertrophied hearts using our software is more accurate than measurements made by conventional TDI procedures.

\section{Methods}

\section{Study Patients}

The study was conducted prospectively from October 2000 to August 2001 in 10 hypertensive patients ( 8 men, 2 women; mean age 56 \pm 7 years, range $44-63$ years) with LV hypertrophy (end-diastolic LV posterior wall thickness $\geq 12 \mathrm{~mm}$ ) and 10 normal controls ( 7 men, 3 women; mean age $54 \pm 5$ years, range $46-68$ years) who underwent Doppler echocardiography for clinical purposes, but who proved not to have cardiovascular disease. All hypertensive patients had their blood pressure controlled by antihypertensive medication, including diuretics, $\beta$-blockers, calcium channel antagonists, angiotensin-converting enzyme inhibitors and/or angiotensin II receptor blockers. All patients were asymptomatic and had no evidence of LV systolic dysfunction or regional asynergy on 2-dimensional (D) echocardiography.

The controls had presented with various complaints, including chest pain, dyspnea, heart murmurs, and arrhythmia, and were evaluated by phonocardiography, routine echocardiography, and/or cardiac catheterization as indi- 
cated. None demonstrated evidence of clinically significant cardiovascular disease. None of the controls had a medical history of systemic disease such as hypertension or diabetes. The test procedures were explained in detail, and informed consent was obtained from each subject.

\section{Study Protocol}

Conventional MVP and MVG (Fig 1) TDI was performed using the commercially available Toshiba SSA390A with a $3.75 \mathrm{MHz}$ probe (Toshiba Corp, Tokyo, Japan). As previously reported, the images showing the motion velocity of the LV posterior wall were superimposed on the M-mode echocardiographic image (Left and Top of Fig 1). The maximal velocity range was established so that the motion velocity would not be saturated $(-14.9$ to 14.9 or -10.8 to $10.8 \mathrm{~mm} / \mathrm{s})$. The beam directions were set to be perpendicular to the LV posterior wall. As in the display of blood flow, the wall motion toward the transducer was coded as red and wall motion away from the transducer was coded as blue. Amplitudes of velocity in each direction were displayed in 200-level color intensity. The time and direction resolutions of the wall motion velocity recorded using this apparatus were approximately $2 \mathrm{~ms}$ and $0.4 \mathrm{~mm}$, respectively. Color-coded and conventional M-mode images were both stored on magneto-optical disks. The color mode can obtain velocity information alone by switching the display mode while the image is kept frozen. Conventional mode was recorded simultaneously to identify the epicardial borders of the LV posterior wall using tissue Doppler M-mode imaging. These images were analyzed using a personal computer (Power PC 7500/100, Apple Computer Inc, Cupertino, CA, USA) and prototype software (Heart Ver 1.4.7, Toshiba Corp). Each velocity was determined from the intensity of the coded red or blue image. The positions of the endocardium and epicardium were determined on the conventional M-mode echocardiogram by manual tracing (Left and Bottom of Fig 1).

Using tissue Doppler M-mode imaging, we manually selected several points in the LV posterior wall and interpolated between the points by using the spline function along both the endocardial and epicardial borders to extract the information about the myocardial velocities. Subsequently, the velocity data between the endocardial and epicardial borders were extracted, and the MVP between the endocardium and epicardium was obtained (Right of Fig 1). The MVG was defined as the slope of a linear regression of the MVP along each M-mode scan line throughout the myocardium. Peak MVGs were defined as the maximum values of velocity gradient during systole and early diastole. By coding the MVG red for a positive gradient and blue for a negative gradient, we reconstructed an MVG image for each tissue Doppler image. The peak systolic and early diastolic wall motion velocity and the MVG during 3 consecutive beats were averaged. The MVP contour at the phase of the maximum MVG was compared between the controls and hypertensive group.

Averaged MVP and $M V G$ (Fig 2) Prototype software (PowerView, Toshiba Corp) was developed to accurately quantify the 2-D color-coded transmural velocity distribution on the parasternal LV short-axis images. This software includes 2 special features. First, after setting the hypothetical center of LV contraction, the velocity data were corrected by the actual Doppler angle of incidence for each pixel in the radial direction (Top and Left, Fig 2). A 2-D region of interest (ROI) (maximum area of $50 \mathrm{~mm}$ in the
Table 1 Clinical and Echocardiographic Characteristics of Control and Hypertensive Patients

\begin{tabular}{lcc}
\hline \hline & Control $(n=10)$ & Hypertensive $(n=10)$ \\
\hline Age (years) & $54 \pm 5$ & $56 \pm 7$ \\
F/M & $3 / 7$ & $2 / 8$ \\
HR $($ beats $/ \mathrm{min})$ & $72 \pm 10$ & $69 \pm 8$ \\
SBP $(\mathrm{mmHg})$ & $124 \pm 8$ & $142 \pm 5^{*}$ \\
VSth $(\mathrm{mm})$ & $9.8 \pm 2$ & $18 \pm 4^{*}$ \\
PWth $(\mathrm{mm})$ & $10.0 \pm 0.8$ & $17 \pm 3 *$ \\
E/A & $0.86 \pm 0.41$ & $0.82 \pm 0.35$ \\
\%FS $(\%)$ & $42 \pm 3$ & $40 \pm 7$ \\
\hline
\end{tabular}

$* p<0.0001$ vs control group

$H R$, heart rate; SBP, systolic blood pressure; VSth, end-diastolic ventricular septal thickness; PWth, end-diastolic left ventricular posterior wall thickness; E/A, ratio of peak early diastolic to atrial systolic velocity of the transmitral flow; \%FS, percent fractional shortening of the left ventricle.

radial direction $\times 15 \mathrm{~mm}$ in the lateral direction) was set along the LV posterior wall with the reference point for angle-correction being at the center of LV contraction. The velocities at each point within the ROI were automatically angle-corrected to calculate the velocity component radial to the LV cavity. The radial direction is defined here as the assumed direction of motion. In the plots shown in the Top and Left panel of Fig 2, the broken lines represent the observed velocity distribution from lines $\mathrm{X}_{1}$ to $\mathrm{Xn}$ at $0.36 \mathrm{~mm}$ intervals, and the solid lines represent the angle-corrected velocity distribution for each radial direction ( $\mathrm{X}_{1}$ to $\mathrm{Xn}$ ) in the ROI. Next, the velocities of $\mathrm{X}_{1}$ to $\mathrm{Xn}$ were averaged along the lateral direction (Top and Right of Fig 2). To improve the stability and accuracy of the velocity profile measurement, the myocardial velocities after angle-correction were spatially averaged along the circumference of the LV short-axis images. The velocities were not averaged in the radial direction in order to maintain the spatial resolution needed to detect the regional velocity gradient in the LV myocardium. The lateral average of the velocity profile at the center of the ROI (line $\mathrm{Xn} / 2$ ) was obtained by taking the average of the angle-corrected velocities at the same radial distance along lines $\mathrm{X}_{1}$ to $\mathrm{Xn}$. The Bottom panel of Fig 2 shows the actual measurement of MVP using conventional (blue) and averaged (yellow) methods. The contour of the MVP at the phase of the maximum MVG was compared between the controls and hypertensive group, and measurements obtained by conventional methodology were compared with our methodology.

\section{Statistical Analysis}

Values are expressed as mean \pm SD. Mean values in the control and hypertensive groups were compared by Student's unpaired t-test. On the MVP determined from conventional and averaged methods, Pearson's linear regression analysis was performed to show the degree of correlation between the velocity and distance from the endocardium to epicardium. The comparison of slope of the linear regression line between conventional and averaged methods in each normal and hypertensive patient was made by Student's paired t-test. The difference in correlation coefficients between conventional and averaged methods in the linear regression lines of MVP in the 10 control and 10 hypertensive patients was evaluated by a Fisher's $Z$ transformation followed by Student's paired t-test. A p $<0.05$ was considered statistically significant. 
Table 2 Linear Regression Lines and Coefficients of the Systolic Myocardial Velocity Profile of the Left Ventricular Posterior Wall Using Conventional and Averaged Methods in 10 Controls

\begin{tabular}{ccc}
\hline \hline Case no. & Conventional & Averaged \\
\hline 1 & $y=-7.8 x+9.5$ & $y=-7.0 x+8.8$ \\
& $(r=-0.93, p<0.0001)$ & $(r=-0.98, p<0.0001)$ \\
$y=-6.9 x+7.3$ & $y=-7.1 x+7.5$ \\
3 & $(r=-0.94, p<0.0001)$ & $(r=-0.97, p<0.0001)$ \\
$y=-5.8 x+7.4$ & $y=-6.0 x+7.5$ \\
4 & $(r=-0.91, p<0.0001)$ & $(r=-0.98, p<0.0001)$ \\
& $y=-5.6 x+7.8$ & $y=-5.5 x+8.1$ \\
5 & $(r=-0.95, p<0.0001)$ & $(r=-0.99, p<0.0001)$ \\
& $y=-5.7 x+9.7$ & $y=-6.0 x+9.5$ \\
6 & $(r=-0.89, p<0.0001)$ & $(r=-0.93, p<0.0001)$ \\
& $y=-8.1 x+8.1$ & $y=-7.9 x+8.3$ \\
7 & $(r=-0.90, p<0.0001)$ & $(r=-0.96 p<0.0001)$ \\
& $y=-5.2 x+7.1$ & $y=-5.3 x+6.8$ \\
8 & $(r=-0.89, p<0.0001)$ & $(r=-0.96, p<0.0001)$ \\
& $y=-6.1 x+7.6$ & $y=-6.3 x+8.0$ \\
9 & $(r=-0.95, p<0.0001)$ & $(r=-0.98, p<0.0001)$ \\
& $y=-7.1 x+7.8$ & $y=-7.3 x+8.3$ \\
10 & $(r=-0.96, p<0.0001)$ & $(r=-0.97, p<0.0001)$ \\
& $y=-7.2 x+9.5$ & $y=-7.8 x+9.3$ \\
& $(r=-0.93, p<0.0001)$ & $(r=-0.99, p<0.0001)$ \\
\hline
\end{tabular}

Table 3 Linear Regression Lines and Coefficients of the Early Diastolic Myocardial Velocity Profile of the Left Ventricular Posterior Wall Using Conventional and Averaged Methods in 10 Controls

\begin{tabular}{ccc}
\hline \hline Case no. & Conventional & Averaged \\
\hline 1 & $y=6.5 x-7.2$ & $y=7.0 x-7.7$ \\
& $(r=0.94, p<0.0001)$ & $(r=0.97, p<0.0001)$ \\
2 & $y=6.5 x-7.4$ & $y=6.2 x-7.6$ \\
& $(r=0.94, p<0.0001)$ & $(r=0.98, p<0.0001)$ \\
3 & $y=7.5 x-7.8$ & $y=7.3 x-8.3$ \\
& $(r=0.95, p<0.0001)$ & $(r=0.98, p<0.0001)$ \\
4 & $y=7.4 x-7.8$ & $y=7.5 x-8.1$ \\
& $(r=0.97, p<0.0001)$ & $(r=0.98, p<0.0001)$ \\
5 & $y=7.7 x-8.8$ & $y=7.7 x-8.9$ \\
& $(r=0.92, p<0.0001)$ & $(r=0.98, p<0.0001)$ \\
6 & $y=5.8 x-7.5$ & $y=5.8 x-8.2$ \\
& $(r=0.85, p<0.0001)$ & $(r=0.96, p<0.0001)$ \\
7 & $y=5.8 x-9.6$ & $y=5.6 x-9.1$ \\
& $(r=0.91, p<0.0001)$ & $(r=0.96, p<0.0001)$ \\
8 & $y=6.1 x-7.6$ & $y=7.7 x-7.9$ \\
& $(r=0.83, p<0.0001)$ & $(r=0.98, p<0.0001)$ \\
9 & $y=6.3 x-6.1$ & $y=6.3 x-6.3$ \\
& $(r=0.94, p<0.0001)$ & $(r=0.99, p<0.0001)$ \\
10 & $y=7.2 x-8.8$ & $y=7.7 x-8.2$ \\
& $(r=0.91, p<0.0001)$ & $(r=0.99, p<0.0001)$ \\
& &
\end{tabular}

\section{Results}

Subject Characteristics

There were no significant differences in age or heart rate between the controls and the hypertensive group (Table 1). Systolic blood pressure was higher in the hypertensive group than in controls, and the thickness of both the enddiastolic ventricular septum and LV posterior wall was greater in the hypertensive group than in controls. The ratio of peak early diastolic to atrial systolic velocity of the transmitral flow and the percent LV fractional shortening were similar in the both groups.

\section{MVP}

Linear regression lines of the MVP in the LV posterior wall and their correlation coefficients in the 10 controls and 10 hypertensive patients are shown in Tables 2-5. Mean
Table 4 Linear Regression Lines and Coefficients of the Systolic Myocardial Velocity Profile of the Left Ventricular Posterior Wall Using Conventional and Averaged Methods in 10 Patients With Hypertensive Hypertrophy

\begin{tabular}{lcc}
\hline \hline Case no. & Conventional & Averaged \\
\hline 11 & $y=-3.5 x+4.4$ & $y=-3.6 x+4.2$ \\
& $(r=-0.91, p<0.0001)$ & $(r=-0.95, p<0.0001)$ \\
12 & $y=-1.7 x+3.0$ & $y=-1.6 x+2.7$ \\
& $(r=-0.89, p<0.0001)$ & $(r=-0.94, p<0.0001)$ \\
13 & $y=-2.3 x+5.5$ & $y=-2.3 x+5.3$ \\
& $(r=-0.88, p<0.0001)$ & $(r=-0.91, p<0.0001)$ \\
14 & $y=-1.5 x+4.8$ & $y=-1.6 x+4.6$ \\
& $(r=-0.89, p<0.0001)$ & $(r=-0.96, p<0.0001)$ \\
15 & $y=-2.5 x+4.7$ & $y=-2.5 x+4.4$ \\
& $(r=-0.92, p<0.0001)$ & $(r=-0.97, p<0.0001)$ \\
16 & $y=-2.9 x+4.4$ & $y=-2.9 x+4.1$ \\
& $(r=-0.85, p<0.0001)$ & $(r=-0.94, p<0.0001)$ \\
17 & $y=-2.1 x+3.6$ & $y=-2.1 x+3.3$ \\
& $(r=-0.92, p<0.0001)$ & $(r=-0.97, p<0.0001)$ \\
18 & $y=-1.3 x+4.1$ & $y=-1.1 x+3.9$ \\
& $(r=-0.92, p<0.0001)$ & $(r=-0.93, p<0.0001)$ \\
19 & $y=-3.0 x+2.9$ & $y=-2.7 x+2.5$ \\
& $(r=-0.89, p<0.0001)$ & $(r=-0.97, p<0.0001)$ \\
20 & $y=-2.5 x+4.4$ & $y=-2.4 x+4.2$ \\
& $(r=-0.89, p<0.0001)$ & $(r=-0.97, p<0.0001)$ \\
& &
\end{tabular}

Table 5 Linear Regression Lines and Coefficients of the Early Diastolic Myocardial Velocity Profile of the Left Ventricular Posterior Wall Using Conventional and Averaged Methods in 10 Patients With Hypertensive Hypertrophy

\begin{tabular}{lcc}
\hline \hline Case no. & Conventional & Averaged \\
\hline 11 & $y=2.5 x-4.5$ & $y=2.0 x-4.4$ \\
& $(r=0.87, p<0.0001)$ & $(r=0.99, p<0.0001)$ \\
12 & $y=2.5 x-5.2$ & $y=2.7 x-4.9$ \\
& $(r=0.89, p<0.0001)$ & $(r=0.97, p<0.0001)$ \\
13 & $y=2.7 x-4.2$ & $y=2.7 x-3.8$ \\
& $(r=0.93, p<0.0001)$ & $(r=0.99, p<0.0001)$ \\
14 & $y=1.5 x-2.8$ & $y=1.4 x-2.5$ \\
& $(r=0.91, p<0.0001)$ & $(r=0.99, p<0.0001)$ \\
15 & $y=2.9 x-4.9$ & $y=2.6 x-4.4$ \\
& $(r=0.90, p<0.0001)$ & $(r=0.99, p<0.0001)$ \\
16 & $y=2.7 x-4.5$ & $y=2.7 x-4.8$ \\
& $(r=0.91, p<0.0001)$ & $(r=0.99, p<0.0001)$ \\
17 & $y=2.1 x-4.6$ & $y=2.1 x-4.4$ \\
& $(r=0.92, p<0.0001)$ & $(r=0.99, p<0.0001)$ \\
18 & $y=2.7 x-4.2$ & $y=2.3 x-3.7$ \\
& $(r=0.93, p<0.0001)$ & $(r=0.98, p<0.0001)$ \\
19 & $y=1.4 x-4.3$ & $y=1.4 x-4.1$ \\
& $(r=0.92, p<0.0001)$ & $(r=0.98, p<0.0001)$ \\
20 & $y=1.9 x-5.5$ & $y=1.8 x-5.0$ \\
& $(r=0.82, p<0.0001)$ & $(r=0.96, p<0.0001)$ \\
& &
\end{tabular}

systolic and early diastolic endocardial wall motion velocities were higher in controls (conventional: $8.2 \pm 1.0 \mathrm{~cm} / \mathrm{s}$ and $-7.9 \pm 0.9 \mathrm{~cm} / \mathrm{s}$, respectively; averaged: $8.2 \pm 0.8 \mathrm{~cm} / \mathrm{s}$ and $-8.0 \pm 0.8 \mathrm{~cm} / \mathrm{s}$, respectively) than in the hypertensive group (conventional: $4.2 \pm 0.8 \mathrm{~cm} / \mathrm{s}$ and $-4.5 \pm 0.7 \mathrm{~cm} / \mathrm{s}$, respectively; averaged: $3.9 \pm 0.9 \mathrm{~cm} / \mathrm{s}$ and $-4.2 \pm 0.7 \mathrm{~cm} / \mathrm{s}$, respectively) (all $p<0.0001)$. The correlation coefficients of the linear regression line of averaged MVP in all control and hypertensive patients were higher than the conventional MVP [systolic MVP in controls (Table 2), $\mathrm{p}<0.000003$; early diastolic MVP in controls (Table 3 ), $\mathrm{p}<0.000006$; systolic MVP in the hypertensive group (Table 4), $\mathrm{p}<0.00006$; early diastolic MVP in the hypertensive group (Table 5), $\mathrm{p}<0.0000008$ ] (Figs 3,4). 
Systolic MVP (case 10)

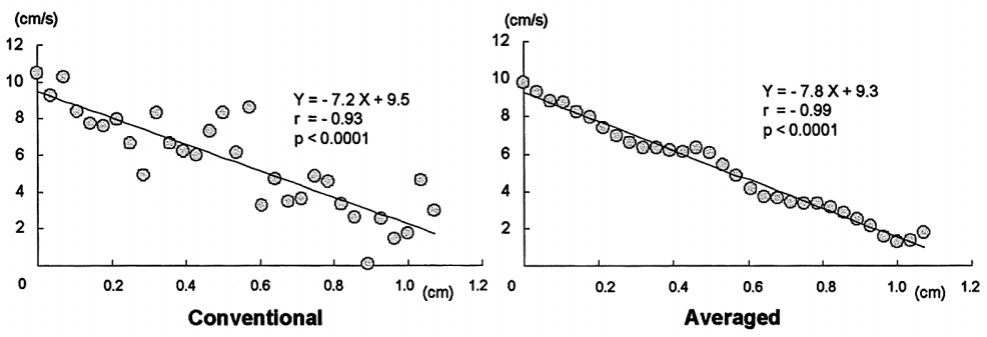

Early diastolic MVP (case 10)

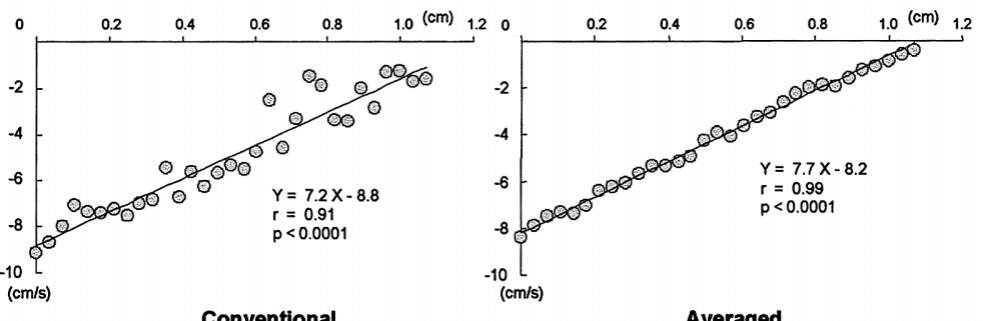

Systolic MVP (case 15)
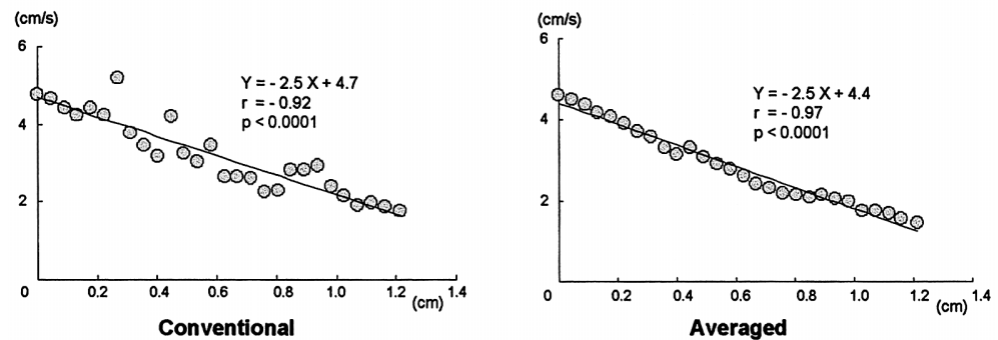

Early diastolic MVP (case 15)

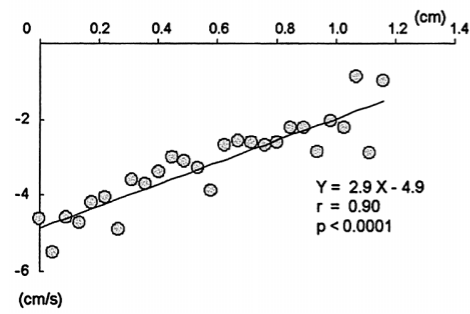

Conventional

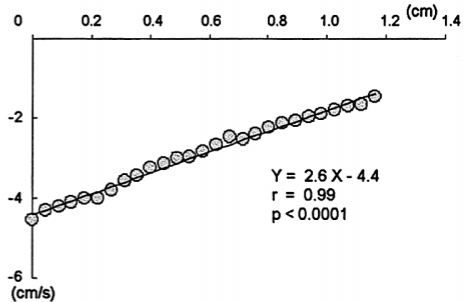

Averaged
Fig 3. Systolic and early diastolic myocardial velocity profiles (MVPs) of the left ventricular posterior wall using conventional and averaged methods in a normal control (case 10).
Table 6 Comparisons of Peak Systolic and Early Diastolic Myocardial Velocity Gradients (MVGs) of the Left Ventricular Posterior Wall Between the Control and Hypertensive Groups

\begin{tabular}{lcc}
\hline \hline & $\begin{array}{c}\text { Conventional } \\
\text { method }\end{array}$ & $\begin{array}{c}\text { Averaged } \\
\text { method }\end{array}$ \\
\hline Control & & \\
Systolic MVG & $-6.6 \pm 1.0$ & $-6.6 \pm 0.9$ \\
Early diastolic MVG & $6.7 \pm 0.7$ & $6.9 \pm 0.8$ \\
Hypertensive & & \\
Systolic MVG & $-2.3 \pm 0.7^{*}$ & $-2.3 \pm 0.7^{+}$ \\
Early diastolic MVG & $2.3 \pm 0.5^{\#}$ & $2.2 \pm 0.5^{*}$ \\
\hline
\end{tabular}

$*_{p}<0.0001$ vs systolic MVG calculated by conventional method in controls. ${ }^{\#} p<0.0001$ vs early diastolic MVG calculated by conventional method in controls. ${ }^{+} p<0.0001 \mathrm{vs}$ systolic MVG calculated by averaged method in controls. ${ }^{\ddagger} p<0.0001$ vs early diastolic MVG calculated by averaged method in controls.

\section{Peak $M V G$}

Mean peak systolic and early diastolic MVGs in the LV posterior wall in controls and the hypertensive group are shown in Table 6. The peak systolic and early diastolic MVGs calculated by the conventional and averaged methods were similar in the controls and the hypertensive group. The peak systolic and early diastolic MVGs were lower in the hypertensive group than in controls.

\section{Assessment of Reproducibility}

Interobserver variabilities of measurements of MVPs and MVGs using conventional and averaged methods (15-19\% and 7-9\%, respectively) were calculated as the difference in 2 measurements of the same subject by 2 different observers divided by the mean value. Intraobserver variabilities using both the methods $(13-15 \%$ and $6-8 \%$, respectively) also was calculated as the difference in 2 measurements of the same subject by a single observer 
divided by the mean value.

\section{Discussion}

This study demonstrates that averaged systolic and early diastolic MVP and MVG in the LV posterior wall of normal and hypertrophied hearts are superior in terms of stability and reproducibility to those recorded by conventional methodology.

Previously, regional LV systolic and diastolic function were evaluated using changes between the endocardium and epicardium on digitized M-mode images; that is myocardial thickening or thinning! 13,14 However, this procedure has some disadvantages, such as errors of measurement because of manual tracing and the difficulty in determining the respective peak values accurately. In recent years, the widespread use of pulsed TDI has facilitated the quantitative evaluation of the LV wall motion velocity 1,4 Because variables obtained by pulsed TDI are not influenced by preload ${ }^{3-5}$ this method is now widely used in clinical settings to evaluate LV diastolic function. Previous studies using pulsed TDI have reported that systolic and early diastolic LV wall motion velocities decrease with age, even in normal subjects 15,16 and that they decrease further in patients with hypertrophic cardiomyopathy, even in the non-hypertrophied regions and when LV pump function is normal! ${ }^{7}$ However, indices obtained by pulsed TDI are affected by the overall heart motion.

Therefore, Fleming et $\mathrm{al}^{18}$ and Uematsu et $\mathrm{al}^{7}$ attempted to evaluate LV wall motion abnormalities quantitatively using color-coded TDI that facilitates the real time and 2-D measurement of wall motion velocity. These investigators measured motion velocities of the rapidly moving endocardium and relatively slow-moving epicardium to calculate the MVG using parasternal LV short-axis images, and reported that regional myocardial function can be evaluated in greater detail using color-coded TDI. Other studies using color-coded TDI also have demonstrated that systolic and early diastolic MVG in the LV wall decrease with age, even in normal subjects, 19 and that the magnitude of the decrease in systolic and early diastolic MVG is markedly higher in patients with hypertrophic cardiomyopathy than in athletes or in patients with hypertensive hypertrophy? ${ }^{20}$ Because color-coded TDI is minimally influenced by the overall heart motion? those previous studies have emphasized that regional LV myocardial function can be evaluated accurately by color-coded TDI even when ventricular septal motion is abnormal, as it is in patients with atrial septal defects? ${ }^{21}$

Thus, TDI provides important information for evaluating regional LV myocardial function in normal and hypertrophied hearts. Based on the hypothesis that the velocity distribution in the myocardium changes linearly, the MVG is expressed by conventional color-coded TDI as the slope of the regression line of changes in motion velocity between the endocardium and epicardium ? $^{-9}$ On the other hand, patients with hypertrophic cardiomyopathy showing nonhomogeneous abnormalities in the LV myocardium and patients with cardiac amyloidosis characterized by granular sparkling, may not have a uniform velocity distribution in the myocardium, and some studies have suggested that such findings are characteristic of the diseased myocardium?,11

This new TDI procedure for obtaining an averaged MVP is characterized by the establishment of a ROI in the LV myocardium after determining the center of the LV cavity using an LV short-axis image as the reference point for angle correction.11,12 During systole and early diastole, a number of MVPs were generated radial to the ROI between the endocardium and epicardium by serially averaging the values in the myocardium from the endocardium to the epicardium circumferentially. This study showed that (1) the MVP recorded by this new TDI procedure is superior in terms of stability and reproducibility to values obtained by conventional TDI procedures; (2) the velocity distribution in normal and hypertensive hypertrophied LV myocardium recorded by our TDI procedure changes linearly, and its correlation coefficient is higher than that obtained by conventional TDI procedures; and (3) systolic and early diastolic MVG in the hypertensive hypertrophied myocardium is lower than that in the normal myocardium, even though the LV myocardial profile in the hypertensive hypertrophied myocardium retains its linear regression. These findings suggest that MVG can be accurately obtained by measuring the averaged MVP, and that the histologic characteristics of the hypertrophied or ischemic myocardium can be also determined by this method? ${ }^{2-25}$

\section{Study Limitations}

Despite the advantage of evaluating regional LV systolic and diastolic function using averaged MVP and peak MVG, TDI has intrinsic technical limitations. As in echocardiographic examination, it cannot accurately reflect wall motion velocity if the direction of the beam is perpendicular to the direction of wall motion (eg, the upper medial and upper lateral ventricular walls).

Furthermore, the center of LV contraction was determined manually by the observers' estimation in the present study, which is potentially a problem in patients with regional LV asynergy.

\section{References}

1. Isaaz K, Thompson A, Ethevenot G, Cloez JL, Brembilla B, Pernot C. Doppler echocardiographic measurement of low velocity motion of the left ventricular posterior wall. Am J Cardiol 1989; 64: 66-75.

2. Yamazaki N, Mine Y, Sano A, Hirama M, Miyatake K, Yamagishi $\mathrm{M}$, et al. Analysis of ventricular wall motion using color-coded tissue Doppler imaging system. Jpn J Appl Phys 1994; 33: 3141-3146.

3. Garcia MJ, Rodriguez L, Ares M, Griffin BP, Thomas JD, Klein AL. Differentiation of constrictive pericarditis from restrictive cardiomyopathy: Assessment of left ventricular diastolic velocities in longitudinal axis by Doppler tissue imaging. J Am Coll Cardiol 1996; 27: $108-114$.

4. Oki T, Tabata T, Yamada H, Wakatsuki T, Shinohara H, Nishikado A, et al. Clinical application of pulsed Doppler tissue imaging for assessing abnormal left ventricular relaxation. Am J Cardiol 1997; 79: $921-928$.

5. Nagueh SF, Middleton KJ, Kopelen HA, Zoghbi WA, Quinones MA. Doppler tissue imaging: A noninvasive technique for evaluation of left ventricular relaxation and estimation of filling pressures. J Am Coll Cardiol 1997; 30: 1527-1533.

6. Onose Y, Oki T, Yamada H, Manabe K, Kageji Y, Matsuoka M, et al. Effect of cilnidipine on left ventricular diastolic function in hypertensive patients as assessed by pulsed Doppler echocardiography and pulsed tissue Doppler imaging. Jpn Circ J 2001; 65: 305-309.

7. Uematsu M, Miyatake K, Tanaka N, Matsuda H, Sano A, Yamazaki $\mathrm{N}$, et al. Myocardial velocity gradient as a new indicator of regional left ventricular contraction: Detection by a two-dimensional tissue Doppler imaging technique. J Am Coll Cardiol 1995; 26: 217-223.

8. Gorcsan J, Gulati VK, Mandarino WA, Katz WE. Color-coded measures of myocardial velocity throughout the cardiac cycle by tissue Doppler imaging to quantify regional left ventricular function. Am Heart J 1996; 131: $1203-1213$.

9. Yamada H, Oki T, Tabata T, Mishiro Y, Abe M, Onose Y, et al. Assessment of the systolic left ventricular myocardial velocity profile and gradient using tissue Doppler imaging in patients with hypertrophic cardiomyopathy. Echocardiography 1999; 16: 775-783.

10. Honma H, Kusama Y, Matsuzaki T, Ohno T, Nishigaki R, Munakata 
$\mathrm{K}$, et al. Resting ultrasonic tissue characterization and dobutamine stress echocardiography for prediction of functional recovery in chronic left ventricular ischemic dysfunction. Jpn Circ J 2001; 65: $381-388$.

11. Tabata T, Oki T, Tanaka H, Onose Y, Matsuoka M, Tada T, et al. Myocardial characterization of cardiac amyloidosis using myocardial velocity profile: Comparison with hypertensive heart disease (abstract). Circulation 2001; 104: II-756.

12. Yamada H, Oki T, Kanda R, Abe Y, Nabatame T. Accurate measurement of transmural myocardial velocity profile using tissue Doppler imaging in normal subjects and patients with left ventricular hypertrophy (abstract). J Am Coll Cardiol 2001; 37: 401A.

13. St John Sutton MG, Tajik AJ, Gibson DG, Brown DJ, Seward JB, Giuliani EF. Echocardiographic assessment of left ventricular filling and septal and posterior wall dynamics in idiopathic hypertrophic subaortic stenosis. Circulation 1978; 57: 512-520.

14. Friendman M, Sahn D, Birris H, Allen H, Goldberg S. Computerized echocardiographic analysis to detect abnormal systolic and diastolic left ventricular function in children with aortic stenosis. Am J Cardiol 1979; 44: 478-486.

15. Yamada H, Oki T, Mishiro Y, Tabata T, Abe M, Onose Y, et al. Effect of aging on diastolic left ventricular myocardial velocities measured by pulsed tissue Doppler imaging in healthy subjects. J Am Soc Echocardiogr 1999; 12: 574-581.

16. Onose Y, Oki T, Mishiro Y, Yamada H, Abe M, Manabe K, et al. Influence of aging on systolic left ventricular wall motion velocities along the long and short axes in clinically normal patients determined by pulsed tissue Doppler imaging. J Am Soc Echocardiogr 1999; 12: $921-926$

17. Oki T, Tabata T, Yamada H, Manabe K, Fukuda K, Abe M, et al. Difference in systolic motion velocity of the left ventricular posterior wall in patients with asymmetric septal hypertrophy and prior anteroseptal myocardial infarction: Evaluation by pulsed tissue Doppler imaging. Jpn Heart J 1998; 39: 163-172.
18. Fleming AD, Xia X, McDicken WN, Sutherland GR, Fenn L. Myocardial velocity gradient by Doppler imaging. Br J Radiol 1994; 67: 679-688.

19. Palka P, Lange A, Fleming AD, Fenn LN, Bouki KP, Shaw TRD, et al. Age-related transmural peak mean velocities and peak velocity gradient by Doppler myocardial imaging in normal subjects. Eur Heart J 1996; 17: 940-950.

20. Palka P, Lange A, Fleming AD, Donnelly JE, Dutka DP, Starkey IR, et al. Differences in myocardial velocity gradient measured throughout the cardiac cycle in patients with hypertrophic cardiomyopathy, athletes and patients with left ventricular hypertrophy due to hypertension. J Am Coll Cardiol 1997; 30: 760-768.

21. Uematsu M, Nakatani S, Yamagishi M, Matsuda H, Miyatake K. Usefulness of myocardial velocity gradient derived from two-dimensional tissue Doppler imaging as an indicator of regional myocardial contraction independent of translational motion assessed in atrial septal defect. Am J Cardiol 1997; 79: 237-241.

22. Shan K, Bick RJ, Poindexter BJ, Shimoni S, Letsou GV, Reardon $\mathrm{MJ}$, et al. Relation of tissue Doppler derived myocardial velocities to myocardial structure and beta-adrenergic receptor density in humans. J Am Coll Cardiol 2000; 36: 891-896.

23. Dutka DP, Donnelly JE, Palka P, Lange A, Nunez DJR, Nihoyannopoulos P. Echocardiographic characterization of cardiomyopathy in Friedreich's ataxia with tissue Doppler echocardiographically derived myocardial velocity gradients. Circulation 2000; 102: $1276-1282$.

24. Derumeaux G, Loufoua J, Pontier G, Cribier A, Ovize M. Tissue Doppler imaging differentiates transmural from nontransmural acute myocardial infarction after reperfusion therapy. Circulation 2001; 103: $589-596$.

25. Matsuoka M, Oki T, Mishiro Y, Yamada H, Tabata T, Wakatsuki T, et al. Early systolic mitral annular motion velocities responses to dobutamine infusion predict myocardial viability in patients with previous myocardial infarction. Am Heart J 2002; 143: 552-558. 